\title{
Ferramenta digital voltada a prevenção do suicídio no Rio Grande do Sul
}

\author{
Marcos Vinnicius Martins \\ Instituto Federal de Educação, Ciência e \\ Tecnologia Farroupilha \\ São Borja, Brasil \\ gronytzki@gmail.com
}

\begin{abstract}
Resumo-Este artigo traz informações relevantes sobre o cenário do Rio Grande do Sul relacionado com as questões pertencentes ao fenômeno do suicídio. Informa dados levantados em uma pesquisa local realizada com profissionais da área da saúde, onde buscou-se informações e subsídios que possibilitem o desenvolvimento de uma ferramenta digital voltada aos dispositivos móveis, com o objetivo proporcionar materiais de promoção à vida, assistência e apoio aos usuários. Aplicando assim, a tecnologia como ferramenta de prevenção ao suicídio, participando e apoiando iniciativas públicas já existentes que tenham foco na redução do número de óbitos por esta causa.
\end{abstract}

Palavras-chave: Suicídio; Ferramenta Digital de Prevenção; Rio Grande do Sul

\section{INTRODUÇÃO}

De acordo com o Boletim de Vigilância Epidemiológica [1] provocar o fim da própria vida está entre as principais causas de morte no mundo, onde a cada40 segundos uma pessoa tira a própria vida, sendo o suicídio a quarta maior causa de morte entre jovens de 15 a 29 anos [2].

O governo do estado do Rio Grande do Sul (RS), alinhado às orientações da Organização Mundial de Saúde (OMS) [3] reconhecendo a necessidade de ações multidisciplinares e intersetoriais, instituiu através do decreto número 53.361 de 22 de dezembro de 2016 o Comitê Estadual de Promoção da Vida e Prevenção do Suicídio. Ricardo Barros ex ministro da saúde do Brasil, conceitua em seu texto de apresentação contido na Agenda de Ações Estratégicas [4], que a prevenção é uma prioridade, e esta representa um desafio para a saúde pública, onde espera-se que a publicação da agenda fortaleça ações de vigilânciae prevenção e agregue novos atores para sua implementação.

Indo de acordo com o governo do estado e as estratégias de prevenção propostas, tem-se como objetivo desenvolver uma ferramenta digital para dispositivos móveis que proporcione materiais de promoção à vida, assistência e apoio aos usuários, colaborando ativamente na prevenção do suicídio no estado gaúcho, aplicando desta forma a tecnologia voltada a dispositivos móveis como uma ferramenta de prevenção ao suicídio.

Uma pesquisa bibliográfica mostra-se imprescindível neste tipo de desenvolvimento, possibilitando compreender o cenário em que a ferramenta será produzida, quais os tipos de dados e como as informações poderão ser manipuladas dentro da mesma. Assim como, descobrir junto de

\author{
Luciéli Tolfo Beque Guerra \\ Instituto Federal de Educação, Ciência e \\ Tecnologia Farroupilha \\ São Borja, Brasil \\ lucieli.beque@iffarroupilha.edu.br
}

profissionais da saúde, por meio de conversas e entrevistas quais os principais recursos e funcionalidades a aplicação necessita ter para dar o devido suporte a seu público alvo.

\section{SUICÍDIO}

Conforme as definições feitas no documento Suicídio, Informando para prevenir [5], o suicídio pode ser definido como um ato deliberado executado pelo próprio individuo, cuja intenção seja a morte, de forma consciente e intencional, mesmo que ambivalente, usando um meio que ele acredita ser letal. Existe também o chamado comportamento suicida: os pensamentos, os planos e a tentativa de suicídio. A Figura 1 [5] ilustra a prevalência desse comportamento suicida na população brasileira ao longo da vida, mostrando que $17 \%$ das pessoas no Brasil, pensaram em algum momento em tirar a própria vida.

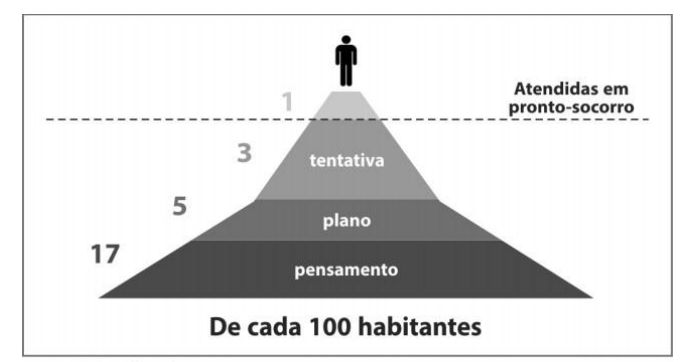

Figura 1. Comportamento suicida ao longo da vida no Brasil

De acordo com a Associação Brasileira de Psiquiatria [5] o suicídio é um comportamento com determinantes multifatoriais e resultado de uma complexa integração de fatores psicológicos e biológicos, inclusive genéticos, culturais e socioambientais. Dessa forma, deve ser considerado como o desfecho de uma série de fatores que se acumulam na história do indivíduo, não podendo ser considerado de forma casual e simplista apenas a determinados acontecimentos pontuais da vida do sujeito trata-se da consequência final de um processo complexo.

\section{A. O Impacto do Suicídio no Mundo}

O suicídio é um fenômeno que ocorre em todas as regiões do mundo. Estima-se que anualmente mais de $80 \mathrm{mil}$ pessoas morrem por suicídio [2]. As taxas vêm aumentando globalmente, até o ano de 2020 poderá ocorrer um incremento de $50 \%$ na incidência de mortes por esta causa em todo o 
mundo, sendo que o número de vidas perdidas ultrapassa o número de mortes decorrentes de homicídio e guerra combinados [5]. Cada morte de adulto cometida corresponde a até vinte tentativas, entre seis a dez pessoas são afetadas diretamente pela perda, com prejuízos emocionais, sociais ou econômicos. Este fenômeno impacta não apenas os sobreviventes, familiares e pessoas próximas a vítima, mas também a comunidade em geral [1].

\section{B. O Impacto do Suicídio no Brasil}

No Brasil os dados apresentados por uma pesquisa realizada com o Sistema de Informação Sobre Mortalidade [2] trouxeram informações alarmantes, em média 10 mil pessoas tiraram a própria vida no ano de 2015 representando a taxa bruta de 5.5/100 mil habitantes. Sabe- se que entre os homens com a faixa etária entre 15 e 29 anos é a quarta maior causa de morte, e entre as mulheres da mesma faixa é a oitava maior causa. O país registrou aumento na taxa de mortalidade por este tipo de fenômeno, quando analisado esta taxa por 100 mil habitantes, constatouse que no ano de 2011 o número de óbitos foi 10.490 , já em 2015 este número aumentou para 11.736 representando um preocupante aumento no número de óbitos.

\section{O Impacto do Suicídio no Rio Grande do Sul}

No RS a taxa de óbitos tem sido em média quase duas vezes maior do que a brasileira, o que representa uma média de três mortes a cada dia. No ano de 2016 foram registrados 1.166 óbitos correspondendo a uma taxa de 11,0 por 100,000 habitantes. Este fenômeno social é reconhecido como um grave problema de saúde pública de acordo com os dados do Boletim de Vigilância Epidemiológica de Suicídio elaborado pela Secretaria da Saúde do estado [1].

\section{Os Caminhos Para a Prevenção}

O suicídio é talvez a forma mais trágica de alguém terminar a própria vida. A maioria das pessoas que consideram esta possibilidade são ambivalentes, ou seja, elas não estão certas se querem realmente terminar sua vida [3]. O suicídio demanda nossa atenção, mas sua prevenção e controle, infelizmente, não são tarefas fáceis, envolvem toda uma série de atividades, que variam desde as melhores condições possíveis no processo de criação das crianças e dos jovens, até o controle dos fatores de risco ambientais [3]. Este fenômeno ainda é cercado de desconhecimento, em função de ser um tabu na sociedade, o estigma em relação ao tema impede a procura de ajuda muitas vezes, mas ainda que o cenário seja alarmante, o suicídio pode ser prevenido a partir de uma análise contextual onde é possível compreender as situações de maior risco.

A disseminação apropriada da informação e o aumento da conscientização são alguns dos elementos essenciais para o sucesso de programas de prevenção ao suicídio afirma o Doutor J. M. Bertolote no prefácio do documento Prevenção do Suicídio: Um
Manual para Profissionais da Mídia [3]. Sabe-se que falar de forma responsável sobre o fenômeno opera muito mais como um fator de prevenção do que como fator de risco, podendo contrapor a suas causas [2]. O psiquiatra Jorge Jaber membro fundador e associado da International Society of Addiction Medicine (ISAM) especialista no assunto, também ressalta que o fundamental é dar atenção e escutar aquele que pensa em cometer suicídio, o fato de alguém que tenta suicídio ser escutado por cerca de 20 minutos pode impedir que ele tenha o impulso de cometer o ato, ouvir o suicida salva a vida dele [6].

De acordo com o Boletim Epidemiológico [2] elaborado pelo Ministério da Saúde, intervenções eficientes bem fundamentadas baseadas em evidências e em dados seguros, podem ser aplicadas a determinados grupos e indivíduos para prevenir as tentativas de suicídio e evitar o óbito por essa causa. A mídia desempenha um papel significativo na sociedade atual ao proporcionar uma ampla gama de informações, com isso, os meios de comunicação podem ter um papel ativo na prevenção do suicídio [3].

\section{E. Promoção da Saúde e Cultura de Paz}

A promoção de saúde passou a ganhar destaque no campo da Saúde Pública a partir da década de 1980 [7]. Um dos primeiros documentos que fez referência ao termo da saúde atual, chama-se Carta de Ottawa, onde a expressão promoção de saúde está associada a um conjunto de valores, são eles: qualidade de vida, saúde, solidariedade, democracia, cidadania, desenvolvimento, participação e parceria. Refere-se também a uma combinação de estratégias: ações do Estado (políticas públicas saudáveis), da comunidade (reforço da ação comunitária), de indivíduos (desenvolvimento de capacidades e habilidades pessoais), do sistema de saúde (reorientação do sistema de saúde) e de parcerias intersetoriais [7].

Existe também a chamada Política Nacional de Promoção a Saúde [7] e esta tem como objetivo promover a qualidade de vida e reduzir a vulnerabilidade e riscos à saúde relacionados a seus determinantes e condicionantes modos de viver, condições de trabalho, habitação, ambiente, educação, lazer, cultura, acesso a bens e serviços essenciais. Proporcionar saúde significa, além de evitar doenças e prolongar a vida, assegurar meios e situações que ampliem a qualidade de vida.

Segundo o Ministério da Saúde (MS) [4] a própria concepção de saúde é uma manifestação da cultura de paz, e também é considerada um completo estado de bem-estar físico, mental e social e não meramente a ausência de doença. Essa é uma perspectiva ampla de saúde, que não se restringe ao indivíduo, mas contempla também coletividade, a sociedade e o planeta.

No Brasil, a Constituição Federal determina que a Saúde é um direito de todos e dever do Estado e 
garante a todas as pessoas acesso universal e igualitário às ações e serviços para sua promoção, proteção e recuperação [4]. No nosso país a saúde não é considerada um bem (que pode ser adquirido por aqueles que têm recursos), mas um direito intrínseco de todas as pessoas, não só brasileiros(as) como de qualquer pessoa em território nacional, todo o sistema de saúde no Brasil é construído sobre valores intimamente associados à Cultura de Paz.

\section{F. Iniciativas Públicas Existentes no Brasil}

O Brasil faz parte dos países que têm participação no Plano em Saúde Mental da OMS onde comprometeu-se com a meta global de redução de $10 \%$ da taxa de suicídio. O Ministério da Saúde diante de toda a relevância deste tema, vem desenvolvendo diversas e consistentes ações de prevenção e vigilância, parcerias com gestores e profissionais de saúde, usuários do Sistema Único de Saúde (SUS), instituições de ensino e pesquisa e a sociedade civil, e visando reforçar ações de prevenção, iniciou no ano de 2017 algumas iniciativas públicas, proporcionando diversos tipos de materiais, sendo um dos mais notórios a Agenda de Ações Estratégicas [4]. Com a publicação da agenda espera-se que amplie e fortaleça as ações de vigilância e prevenção, assim como agregue novos atores para sua implementação. Por meio do Plano Nacional de Prevenção ao Suicídio, pretende-se, até o ano de 2020 ampliar e fortalecer as ações de promoção da saúde, vigilância. Conforme mencionado pela Secretária de Saúde do Rio Grande do Sul [1], o governo do estado, reconhece a necessidade de ações multidisciplinares e intersetoriais.

Agindo de acordo com o Plano Nacional de Prevenção do Suicídio e com o objetivo específico da Agenda Estratégica pretende-se apresentar requisitos de software para uma ferramenta digital com o foco na promoção da vida, objetivando a prevenção do suicídio no estado do RS, participando e apoiando iniciativas públicas já existentes, agindo de acordo as ações contidas na Agenda de Ações Estratégicas de [4]:

- Fomentar parcerias com instituições de ensino e pesquisa, profissionais e sociedade civil, para desenvolvimento de estudos relacionados a prevenção do suicídio com foco nas populações e grupos em situação de vulnerabilidade.

- Fomentar projetos pilotos locais para implementar a operacionalização das Diretrizes Nacionais de Prevenção do Suicídio.

- Aprimorar a qualidade da informação por meio de relacionamento de bancos de dados provenientes de diferentes Sistemas de Informação.

Entende-se a necessidade do uso de informações, tanto para o acionamento imediato da rede de atenção, o acompanhamento dos casos e a intervenção precoce e adequada. A Secretaria de Vigilância [2] conceitua que algumas ações podem prevenir a ocorrência e a concretização de novas tentativas de suicídio, além disso, destinar recursos para sua prevenção é um caminho estratégico para preservar e melhorar a qualidade de vida de muitas pessoas.

\section{CENTRO DE VALORIZAÇÃO DA VIDA}

Uma das principais organizações que realizam este serviço de dar suporte gratuito as pessoas que passam por alguma situação relacionada ao o suicídio é o Centro de Valorização da Vida (CVV). Conforme as informações presentes no site oficial da instituição [8], o Centro de Valorização da Vida, foi fundado em São Paulo, em 1962, e é uma associação civil sem fins lucrativos, filantrópica, reconhecida como de Utilidade Pública Federal, desde 1973 presta serviço voluntário e gratuito de apoio emocional e prevenção do suicídio para todas as pessoas que querem e precisam conversar, sob total sigilo e anonimato.

\section{A. Canais de Comunicação do CVV}

A instituição disponibiliza alguns meios de comunicação ao público conforme exibido na Figura 2, atendendo voluntária e gratuitamente todas as pessoas que querem e precisam conversar, sob total sigilo por telefone, e-mail e chat 24 horas todos os dias.

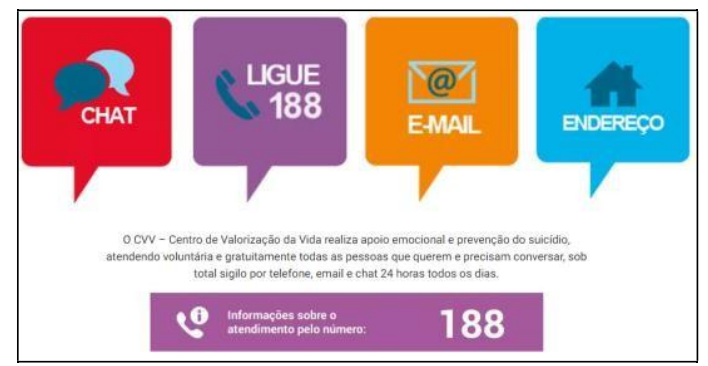

Figura 2. Canais de comunicação do CVV

Dentre os meios de comunicação proporcionados até o momento pela instituição, percebe-se a falta de suporte do seu serviço aos usuários de dispositivos móveis. Sendo esta tecnologia uma das mais utilizadas em todo o Brasil, podendo assim, fazer com que muitas pessoas não utilizem os recursos de assistência que eles oferecem.

\section{A TECNOLOGIA VOLTADA À PREVENÇÃO DO SUICÍDIO}

\section{A. A Comunicação Digital}

O ser humano é um ser social, está sempre interagindo com outras pessoas em seu cotidiano, as conversas cara a cara ainda são importantes para as interações sociais, mas a comunicação online mudou a forma como as pessoas entram em contato umas com as outras [9].

A Internet é a ferramenta que têm permitido esse tipo de evolução da comunicação, e vêm sendo muito 
utilizada no Brasil, como mostra a pesquisa TIC Domicílios 2015 realizada pelo PORTAL BRASIL [10]. Cerca de $58 \%$ da população brasileira usa a internet, o que representa 102 milhões de usuários, sendo que a maioria dos acessos à internet ocorre por meio do celular (89\%), seguido pelo computador de mesa (40\%), computador portátil ou notebook (39\%), tablet $(19 \%)$, televisão $(13 \%)$ e videogame $(8 \%)$. A região Sudeste tem 17,4 milhões de domicílios conectados à internet; no Nordeste são 7 milhões; a região Sul possui 5,4 milhões; o Centro-Oeste tem 2,5 milhões; e no Norte são 1,9 milhão.

De acordo com o relatório publicado pela International Telecommunication Union Place des Nations [11] a rede móvel chegou como uma forma mais penetrante e inclusiva por meio de celulares de baixo custo, recargas pré-pagas e serviços financeiros móveis, transformando a tecnologia móvel em uma ferramenta de desenvolvimento que mudou a forma como as pessoas planejam sua vida, se organizam socialmente, e acessam oportunidades de educação, saúde, negócios e de emprego.

\section{B. Dispositivos Móveis}

De acordo com a definição de TECHOPEDIA [12], um aplicativo móvel é um tipo de software projetado para rodar em um dispositivo móvel, como telefone celular, smartphone ou tablet. Eles geralmente são pequenos e com funções limitadas, devido à quantidade de processamento de um smartphone em relação aos computadores tradicionais, entretanto, os aplicativos móveis geralmente fornecem os mesmos serviços que os acessados nos computadores.

Nos últimos anos a procura por mobilidade aumentou consideravelmente, usuários e empresas de pequeno a grande porte têm procurado tecnologias que atendam todas as suas necessidades diárias e que possam ser utilizadas em qualquer lugar e a qualquer momento. Desta forma, o mercado de dispositivos móveis vem a cada ano se tornando mais poderoso e promissor. Cerca de três bilhões de pessoas já possuem ao menos um dispositivo móvel, e este número vêm aumentando a cada ano [13].

\section{Aplicativos com Foco na Prevenção}

Em relação a utilização de aplicativos moveis pelos usuários, sabe-se que a falta de anonimato é considerada um problema quando este faz parte de uma comunidade onde os profissionais são conhecidos popularmente. $\mathrm{O}$ estigma e a vergonha são considerados barreiras para a busca de ajuda e podem contribuir para que pessoas com comportamento suicida não procurem ajuda no momento em que precisam. Com isso, [14] o apoio anônimo pode superar alguns desses obstáculos e os aplicativos de dispositivos móveis podem fornecer um suporte mais acessível.

Nesse contexto, sabe-se que a população adota e busca cada vez mais aplicativos de celulares para gerenciar sua saúde e rotina de vida. Segundo Larsen [15], 85\% dos jovens nos Estados Unidos possuem smartphone e três quartos deles os utilizam para acessar informações de saúde. Os autores afirmam que em uma pesquisa realizada, $69 \%$ dos entrevistados e $80 \%$ dos que tinham 45 anos de idade ou menos demonstraram desejo em utilizar aplicativos móveis para rastrear sua saúde mental.

Uma pesquisa brasileira [16] investigou o uso de serviços para a população de usuários que tenham 16 anos ou mais em algumas áreas como saúde e educação, e concluiu que em 2015 a população que buscou informações em ao menos uma dessas áreas, foi de $59 \%$. Sendo assim, percebe-se a busca por serviços de atendimento à saúde através de dispositivos móveis, confirmando a necessidade de ampliar a disponibilização dos mesmos à população.

Conforme a Organização Mundial de Saúde [14], as intervenções via aplicativos podem ser úteis em países em desenvolvimento, onde o apoio às pessoas com risco de suicídio pode ser limitado. Desse modo, a organização faz recomendações acerca do uso de dispositivos móveis, considerando estes uma opção para apoio e tratamento das pessoas em risco de suicídio, uma vez que se sabe que o aplicativo de celulares tem potencial de atingir um grande número de pessoas através do uso de dispositivos eletrônicos e que podem ser implementados a um baixo custo [14].

\section{METODOLOGIA}

Buscando a melhor compreensão e entendimento do cenário referente ao suicídio no estado do Rio Grande do Sul uma pesquisa bibliográfica foi realizada em materiais da área da saúde. Também foi realizado o estudo e levantamento das iniciativas públicas já existentes no estado gaúcho que tenham foco na prevenção deste problema.

Esta pesquisa possibilitou compreender o cenário em que a ferramenta será produzida, quais os tipos de dados e como as informações poderão ser manipulados. Junto de profissionais da saúde por meio de conversas e entrevistas foi possível descobrir alguns dos principais recursos e funcionalidades que a ferramenta necessita apresentar, para dar o devido suporte a seu público alvo.

Com base no cenário estudado foram definidos e listados alguns requisitos de software. Com os recursos que se destacaram na pesquisa foram produzidos protótipos de tela visando a demonstração de algumas funcionalidades da ferramenta.

\section{RESUltados}

Baseando-se na pesquisa bibliográfica realizada nos materiais selecionados da área da saúde, que possuíam enfoque em técnicas e maneiras de identificar e prevenir o suicídio, assim como, nas informações coletadas durante a entrevista realizada com uma psicóloga local da saúde, identificou-se em uma conversa inicial alguns recursos e funcionalidades que a aplicação poderia ter para dar o devido suporte a seu público alvo. A Tabela 1 
apresenta essas informações.

TABELA 1. AÇÕES RECOMENDADAS QUE A FERRAMENTA PODE CONTEMPLAR

\begin{tabular}{|c|c|}
\hline & Descrição \\
\hline 01 & Despertar o lado saudável da pessoa. \\
\hline 02 & Fazer orientações motivadoras e saudáveis aos usuários. \\
\hline 03 & Mandar conteúdos periódicos sobre a valorização da vida \\
\hline 04 & $\begin{array}{l}\text { Proporcionar ao usuário um local seguro e sigiloso em } \\
\text { que possa se expressar. }\end{array}$ \\
\hline 05 & $\begin{array}{l}\text { Gerar vinculo constante com o usuário, para que } \\
\text { quando precisar ele lembre que tem o suporte na } \\
\text { ferramenta. }\end{array}$ \\
\hline 06 & $\begin{array}{l}\text { Aos usuários adolescentes, as mensagens motivadoras } \\
\text { podem ter o foco social, de acordo com sua idade, como: } \\
\text { "Ligue para seu amigo mais próximo; abrace seu animal } \\
\text { de estimação; saia, de casa e faça uma caminhada para } \\
\text { relaxar". }\end{array}$ \\
\hline 07 & $\begin{array}{l}\text { Aos usuários adultos, as mensagens motivadoras podem } \\
\text { ter foco familiar, de acordo com sua idade, como: } \\
\text { "Interaja com seus filhos; abrace seu (a) parceiro (a)". }\end{array}$ \\
\hline 08 & $\begin{array}{l}\text { Possibilitar que o usuário se expresse em um momento } \\
\text { especifico, exemplo: “O que você está pensando agora? } \\
\text { o que você está sentindo?". }\end{array}$ \\
\hline 09 & $\begin{array}{l}\text { Possuir um mecanismo de emergência, onde, alguns } \\
\text { telefones de familiares e serviços de apoio ao } \\
\text { suicídio são } \\
\text { acessados de forma rápida e eficiente. }\end{array}$ \\
\hline 10 & Estimular o usuário repensar alguns aspectos de sua vida. \\
\hline 11 & $\begin{array}{l}\text { Estimular o usuário a ressignificar esse momento } \\
\text { em que está passando. }\end{array}$ \\
\hline 12 & $\begin{array}{l}\text { Dar a possibilidade para usuário fazer uma projeção } \\
\text { boa de seu futuro. }\end{array}$ \\
\hline
\end{tabular}

Com base nas informações elencadas na entrevista com a profissional, foi realizado um estudo e proposto alguns requisitos de software que a ferramenta terá quando desenvolvida. Estes requisitos estão disponíveis para os dois tipos de usuários, e podem ser vistos nas Tabelas 2 e 3 .

\section{TABELA 2. REQUISITOS DE SOFTWARE DO USUÁRIO}

\begin{tabular}{|c|l|}
\hline & \multicolumn{1}{|c|}{ Descrição } \\
\hline 01 & Cadastrar-se no aplicativo. \\
\hline 02 & Cadastrar informações de emergência. \\
\hline 03 & $\begin{array}{l}\text { Acessar o mecanismo de emergência e ser direcionado } \\
\text { para falar com os contatos que foram cadastrados e/ou } \\
\text { com número do CVV. }\end{array}$ \\
\hline $\begin{array}{l}\text { Avaliar qualitativamente e/ou escrever um texto sobre } \\
\text { algumas categorias pré-definidas (Saúde, }\end{array}$ \\
$\begin{array}{l}\text { Relacionamento, Trabalho, Estudos) na seção feita para } \\
\text { ele repensar. }\end{array}$ \\
\hline 05 & $\begin{array}{l}\text { Informar objetivos no formato de lista pré-definida com } \\
\text { categorias e/ou escrever texto livre. }\end{array}$ \\
\hline 07 & $\begin{array}{l}\text { Receber notificações e sugestões no aplicativo com base } \\
\text { nas informações cadastradas. } \\
\text { Avaliar qualitativamente, escrever um texto livre, } \\
\text { responder de forma textual algumas perguntas } \\
\text { predeterminadas. }\end{array}$ \\
\hline 08 & $\begin{array}{l}\text { Avaliar conteúdo que recebeu em seu perfil com base } \\
\text { nas informações que recebeu. }\end{array}$ \\
\hline
\end{tabular}

\begin{tabular}{|c|l|}
\hline 09 & $\begin{array}{l}\text { Visualizar como está sua evolução desde que } \\
\text { começou a utilizar a ferramenta. }\end{array}$ \\
\hline 10 & $\begin{array}{l}\text { Receber notificações periódicas sobre a valorização da } \\
\text { vida. }\end{array}$ \\
\hline
\end{tabular}

A Tabela 2 apresenta alguns dos principais requisitos de software que a ferramenta poderá contemplar, estes ao serem desenvolvidos e implementados, representam as funcionalidades que o usuário que necessita de ajuda terá acesso ao utilizar a ferramenta.

TABELA 3. REQUISITOS DE SOFTWARE DO PROFISSIONAL

\begin{tabular}{|c|c|}
\hline & Descrição \\
\hline 01 & Cadastrar-se no aplicativo. \\
\hline 02 & $\begin{array}{l}\text { Disponibilizar conteúdos, podendo ser texto, imagem } \\
\text { ou vídeo. }\end{array}$ \\
\hline 03 & Visualizar seus conteúdos já cadastrados. \\
\hline 04 & $\begin{array}{l}\text { Visualizar estatísticas do número de acesso e das } \\
\text { avaliações feitas pelos usuários de um determinado } \\
\text { conteúdo. }\end{array}$ \\
\hline 05 & Editar suas informações pessoais. \\
\hline
\end{tabular}

A Tabela 3 demonstra os requisitos de software elaborados para o profissional da saúde, estes representam as ações que este usuário poderá fazer ao utilizar a ferramenta. Para fins de demonstração, de maneira visual de alguns dos conceitos do desenvolvimento, dois protótipos de tela foram elaborados utilizando a ferramenta digital open-source chamada Pencil.

O primeiro protótipo da Figura 3 demonstra algumas opções que o usuário poderá realizar ao utilizar o aplicativo.

Esta seção do aplicativo, apresentado na Figura 3, é voltada ao usuário que precisa de ajuda. Nela ele avalia em um determinado tempo como está se sentindo, e informa ao aplicativo que tipo de conteúdo ele tem interesse em receber. O segundo protótipo, apresentado pela Figura 4, demonstra algumas ações que o profissional da saúde poderá interagir ao utilizar a ferramenta.

Esta seção do aplicativo (Figura 4) é voltada ao profissional da saúde que vai elaborar e direcionar os conteúdos e informações contidos na aplicação. Nela, ele pode escolher entre algumas opções de conteúdo como: textos, imagens, vídeos, onde o mesmo escolhe alguns filtros para associar ao seu conteúdo criado.

As interações se darão das seguintes formas: A ferramenta analisa quais os conteúdos foram criados pelos profissionais da saúde e a quais perfis de usuários devem ser direcionados, o usuário que precisa de auxílio, irá interagir por meio de seções interativas com seu assistente pessoal, este irá direcionar para ele conteúdos com o enfoque na valorização da vida, assistência e apoio, conteúdos estes relacionados com a situação atual, baseados em uma avaliação dele durante sua utilização do aplicativo. 


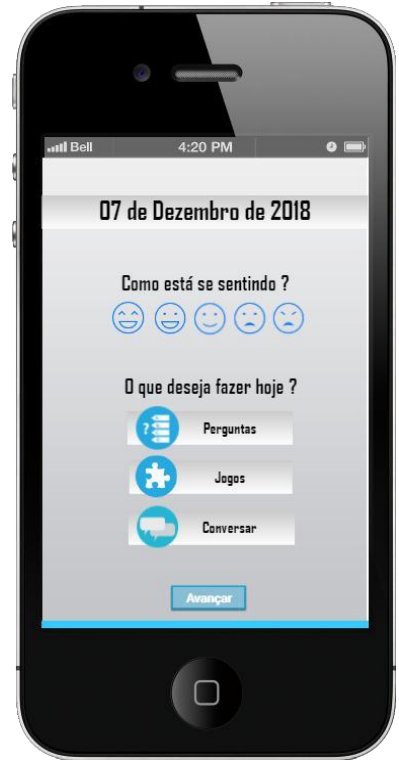

Figura 3. Protótipo de tela, interação do usuário

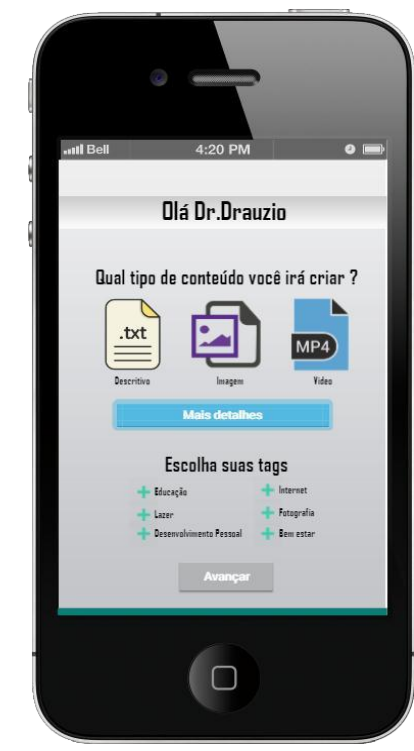

Figura 4. Protótipo de tela, interação do profissional da saúde

\section{CONCLUSÃO}

Tendo o fenômeno do suicídio visto como algo extremamente sério em todo o mundo [5], onde este possui números assustadores nos índices de óbitos brasileiros e é reconhecido oficialmente pela Secretaria de Saúde do Rio Grande do Sul [1] como um grave problema de saúde pública, este artigo buscou através de uma pesquisa substancial, apresentar informações oficiais sobre o cenário gaúcho, sua relação com o fenômeno do suicídio. Trouxe ainda informações conceituais sobre o mesmo, juntamente de algumas políticas públicas e ações que vêm sendo realizadas no Brasil com o foco na prevenção e diminuição do número de obtidos por esta causa.
Para contribuir positivamente na prevenção e agir em conjunto com as iniciativas públicas brasileiras estudadas, foi realizada uma pesquisa com profissionais da área da saúde, mais especificadamente, uma psicóloga. Nesta etapa, foi possível compreender as informações ligadas ao suicídio pelo ponto de vista da profissional, assim como, entender quais requisitos e funcionalidades a aplicação poderá contemplar em sua fase de desenvolvimento, tendo o objetivo de proporcionar ao usuário os melhores recursos de promoção da vida e de prevenção.

A partir de toda a pesquisa que foi realizada até o momento, foi possível compreender o cenário geral relacionado com o suicídio, identificar os recursos que a ferramenta digital poderá contemplar, onde estes foram disponibilizados e organizados em uma tabela. Após feita análise e organização das informações levantadas, foram propostos alguns requisitos de software para serem utilizados no desenvolvimento da ferramenta, também foram propostos protótipos de tela, que trazem algumas das interações que poderão ser realizadas dentro da ferramenta digital de prevenção. No futuro, com o desenvolvimento da ferramenta, será possível aplicar e testar os requisitos propostos por esta pesquisa com os usuários e profissionais da saúde, mensurando a contribuição da ferramenta digital no caminho da prevenção deste grave problema de saúde.

\section{REFERÊNCIAS}

[1] RIO GRANDE DO SUL. Boletim de vigilância epidemiológica de suicídio e tentativa de suicídio. Volume 1, Número 1. Secretaria da saúde. Rio grande do Sul: Porto Alegre, setembro 2018. Disponível em: <www.cevs.rs.gov.br>. Acesso em: novembro, 2018.

[2] BRASIL. Suicídio. Saber, agir e prevenir. Boletim Epidemiológico. Volume 48, número 30. ISSN 2358-9450. Ministério da Saúde, Secretaria de vigilância em Saúde: Brasil, 2017b. Disponível

em:

<http://portalms.saude.gov.br/images/pdf/2017/setembro/ 21/2017- 025-Perfilepidemiologico-das-tentativas-eobitos-por-suicidio-no- Brasil-e-a-rede-de-atencaoasaude.pdf $>$. Acesso em: dezembro,

[3] OMS. Prevenção do Suicídio: Um Manual para Profissionais da Mídia. Organização Mundial da Saúde, Departamento de Saúde Mental: Transtornos Mentais e Comportamentais. Genebra, 2000. Disponível em:

$<$ https://www.who.int/mental_health/prevention/suicide/e $\mathrm{n} /$ suicide prev_media_port.pdf $>$. Acesso em: fevereiro, 2019.

[4] BRASIL. Agenda de Ações Estratégicas para a Vigilância e Prevenção do Suicídio e Promoção da Saúde no Brasil: 2017 a 2020 [recurso eletrônico] / Ministério da Saúde, Secretaria De Atenção À Saúde, Departamento De Ações Programáticas Estratégicas. - Brasília: Ministério da Saúde, 2017a. Disponível em: <https://www.neca.org.br/wpcontent/uploads/cartilha_agendaestrategicapublicada.pdf>. Acesso em: janeiro, 2019.

[5] BRASIL. Suicídio: informando para prevenir. Associação Brasileira de Psiquiatria. Brasília, Brasil, 2014a. Disponível em: <https://www.cvv.org.br/wp- 
content/uploads/2017/05/suicidio_informado_para_preven ir_abp_2 014.pdf >. Acesso em: maio, 2019.

[6] GANDRA, Lana. Campanha vai usar redes sociais para prevenir suicídio. $\quad$ Setembro 2018.

Disponível em:

<http://agenciabrasil.ebc.com.br/saude/noticia/2018-

09/cada-40- segundos-ha-um-suicidio-no-mundo>.

Acesso em: agosto de 2019.

[7] CAMPOS, M. O.; NETO, J. R. Qualidade de Vida: Um Instrumento para Promoção de Saúde. STOA SP, 32(2), 232-240. 2008. Disponível em: http://stoa.usp.br/lislaineaf/files/- 1/19150/qualidade-vidainstrumentopromocao-saude.pdf. Acesso em: junho, 2019.

[8] CVV. Centro de Valorização da Vida. 2019. Disponível em: < https://www.cvv.org.br/>. Acesso em: maio de 2019

[9] PAVAN, Marina. Design e tecnologia: criação de ferramenta de conscientização sobre depressão. 2017. Monografia (Graduação em Design) - Universidade do Vale do Taquari - Univates, Lajeado,

27 nov. 2017. Disponível em: http://hdl.handle.net/10737/1881 Acesso em: junho, 2019.

[10] PORTAL BRASIL. Pesquisa revela que mais de 100 milhões de brasileiros acessam a internet. Disponível em: $<$ http://www.brasil.gov.br/ciencia-e-

tecnologia/2016/09/pesquisa- revela-que-mais-de-100 milhoes-de-brasileiros-acessam-a-internet>. Accesso em: junho, 2019.

[11] ITU. Measuring the Information Society Report. Geneva: International Telecommunication Union, 2016. Disponível em: < https://www.itu.int/en/ITU D/Statistics/Documents/publications/misr2016/MISR2016w4.pdf

>. Acesso em: junho, 2019.

[12] TECHOPEDIA. Mobile Application (Mobile App). Disponívelem:

<https://www.techopedia.com/definition/2953/mobileapplication- mobile-app>. Acesso em: junho, 2019.

[13] NUNES, Fernando. Avaliação de Técnicas e Mecanismos para Entrada e Saída de Informações em Dispositivos Móveis. 2014. 55

f. Trabalho de Curso (Bacharelado em Sistemas de Informação) - Centro Universitário Eurípides de Marília, Fundação de Ensino "Eurípides Soares da Rocha", Marília, $2014 . \quad$ Disponível em https://aberto.univem.edu.br/handle/11077/998. Acesso em: junho, 2019.

[14] BARBOSA, Sarah Soares Funcionalidades para aplicativo de celular à pessoa com comportamento suicida no Brasil. 108 p. 2018. Trabalho de Conclusão de Curso - Curso de Graduação em Enfermagem da Universidade Federal de Santa Catarina, Florianópolis, $2018 . \quad$ Disponível em: <https://repositorio.ufsc.br/handle/123456789/187180 Acesso em: junho, 2019.>

[15] LARSEN, Mark Erik; NICHOLAS, Jennifer; CHRISTENSEN, Helen. A Systematic Assessment of Smartphone Tools for Suicide Prevention. Plos One, [s.1.], v. 11, n. 4, p.1-14, 13 abr. 2016.Public Library of Science (PLoS). Disponível em:

<http://journals.plos.org/plosone/article?id=10.1371/journ al.pone.0 152285>. Acesso em: junho de 2019.

[16] BRASIL. Comitê Gestor da Internet. Pesquisa sobre o uso das tecnologias de informação e comunicação nos domicílios brasileiros [livro eletrônico]: TIC domicílios 2015a. São Paulo, $2015 . \quad 424 p$.

Disponível

em:<http://cetic.br/media/docs/publicacoes/2/TIC_Dom_2 015_LIVRO_ELETRONICO.p>. Acesso em: junho, 2019. 